\title{
MINI-REVIEW
}

\section{Treatment of Fragile X Syndrome with Cannabidiol: A Case Series Study and Brief Review of the Literature}

\author{
Nicole Tartaglia, ${ }^{1}$ Marcel Bonn-Miller, ${ }^{2}$ and Randi Hagerman ${ }^{3, *}$
}

\begin{abstract}
Fragile $X$ syndrome (FXS) is an X-linked dominant disorder caused by a mutation in the fragile $X$ mental retardation 1 gene. Cannabidiol (CBD) is an exogenous phytocannabinoid with therapeutic potential for individuals with anxiety, poor sleep, and cognitive deficits, as well as populations with endocannabinoid deficiencies, such as those who suffer from FXS. The objective of this study was to provide a brief narrative review of recent literature on endocannabinoids and FXS and to present a case series describing three patients with FXS who were treated with oral CBD-enriched (CBD+) solutions. We review recent animal and human studies of endocannabinoids in FXS and present the cases of one child and two adults with FXS who were treated with various oral botanical CBD+ solutions delivering doses of 32.0 to $63.9 \mathrm{mg}$ daily. Multiple experimental and clinical models of FXS combine to highlight the therapeutic potential of CBD for management of FXS. All three patients described in the case series exhibited functional benefit following the use of oral CBD+ solutions, including noticeable reductions in social avoidance and anxiety, as well as improvements in sleep, feeding, motor coordination, language skills, anxiety, and sensory processing. Two of the described patients exhibited a reemergence of a number of FXS symptoms following cessation of CBD+ treatment (e.g., anxiety), which then improved again after reintroduction of CBD+ treatment. Findings highlight the importance of exploring the therapeutic potential of CBD within the context of rigorous clinical trials.
\end{abstract}

Keywords: cannabidiol; CBD; endocannabinoid system; fragile X syndrome; oral solution

\section{Introduction}

Fragile X syndrome (FXS) is an X-linked dominant disorder caused by the expansion of a trinucleotide repeat (CGG)n within the first exon of the fragile $\mathrm{X}$ mental retardation 1 (FMR1) gene, which silences the expression of the fragile X mental retardation protein (FMRP). ${ }^{1}$ The absence of FMRP, an important regulator of translation of many messenger RNAs involved in synaptic plasticity, ${ }^{2}$ leads to substantial intellectual deficits. The condition has been estimated to affect $\sim 1: 4000$ males and 1:7000 females. ${ }^{3-5}$ Although both sexes are susceptible, males with FXS typically exhibit more severe symptoms compared with females because the single $\mathrm{X}$ chromosome in males is usually fully methylated and not producing $\mathrm{FMRP}^{6}$; females typically have some
FMRP expression from the FMR1 gene on their second unaffected X chromosome. ${ }^{7}$ Males with FXS are far more likely than females to exhibit significant intellectual and developmental disabilities (85\% vs. $25 \%){ }^{8}$

Among individuals with the full mutation (i.e., $>200$ CGG repeats), symptoms of FXS vary by age and sex, but often include anxiety (80\%), social avoidance (80\%), stereotyped behaviors (80\%), attention-deficit/ hyperactivity disorder (70\%), autism spectrum disorders $(60 \%)$, intellectual disability ( $85 \%$ of males and $25 \%$ of females), aggression (40\%), disrupted sleep patterns $(40 \%)$, and epilepsy (16\%), as well as macroorchidism (90\% of adults), prominent ears (60\%), long faces $(60 \%)$, soft skin (50\%), and hyperextensible joints (60\% of children). ${ }^{9-11}$ Traditional allopathic treatment of

\footnotetext{
${ }^{1}$ Department of Pediatrics, University of Colorado School of Medicine, Aurora, Colorado.

${ }^{2}$ Zynerba Pharmaceuticals, Devon, Pennsylvania.

${ }^{3}$ Department of Pediatrics, MIND Institute, University of California Davis Medical Center, Sacramento, California.
}

*Address correspondence to: Randi Hagerman, MD, Department of Pediatrics, MIND Institute, University of California Davis Medical Center, Sacramento UC Davis Medical Center, 2825 50th Street, Sacramento, CA 95817, E-mail: rjhagerman@ucdavis.edu

(c) Nicole Tartaglia et al. 2019; Published by Mary Ann Liebert, Inc. This Open Access article is distributed under the terms of the Creative Commons License (http://creativecommons.org/licenses/by/4.0), which permits unrestricted use, distribution, and reproduction in any medium, provided the original work is properly cited. 
these patients may involve medications to address issues with sleep (melatonin and clonidine), anxiety (selective serotonin reuptake inhibitors and benzodiazepines), hyperactivity and deficits in attention (psychostimulants), and seizures (carbamazepine, valproic acid, and lamotrigine). ${ }^{12}$ Yet, for many patients with FXS, the aforementioned pharmacotherapies, when used alone or in combination, have suboptimal efficacy and tolerability, ${ }^{11-13}$ suggesting a persistent unmet medical need for novel, interventional treatment approaches for patients with FXS. Because the clinical abnormalities in these patients (and most recently in children with autism spectrum disorder ${ }^{14}$ ) have been linked, at least in part, with dysregulation of the endocannabinoid system, we briefly review recent research characterizing the involvement of the endocannabinoid system in FXS and present the cases of three individuals (one child and two adults) with FXS who experienced functional benefit after treatment with cannabidiol (CBD)enriched $(\mathrm{CBD}+)$ preparations.

\section{Endocannabinoids and FXS}

The endocannabinoid system consists of receptors in the brain and peripheral tissues that are involved in numerous physiological processes as well as the endocannabinoids, $\mathrm{N}$-arachidonoylethanolamine (anandamide; AEA) and 2-arachidonoylglycerol (2-AG). The endocannabinoids bind to the G-protein-coupled receptors, cannabinoid 1 and $2\left(\mathrm{CB}_{1}\right.$ and $\left.\mathrm{CB}_{2}\right),{ }^{15,16}$ and modulate synaptic transmission throughout the central nervous system. ${ }^{17,18}$ Receptors are distributed throughout the body, with $\mathrm{CB}_{1}$ receptors abundantly expressed in the brain and present at lower concentrations in a variety of peripheral tissues and cells and $\mathrm{CB}_{2}$ receptors expressed primarily in the immune and hematopoietic systems, as well as in the brain, pancreas, and bone. ${ }^{16}$

CBD, the primary, noneuphoric exogenous phytocannabinoid in cannabis, may attenuate the loss of endogenous cannabinoid (i.e., endocannabinoid) signaling observed in preclinical models of FXS, allowing a component of the FMRP deficiency inherent in FXS to be bypassed. Specifically, many abnormalities seen in FXS appear to be rooted in dysregulation of the endocannabinoid pathways in the central nervous system, with a reduction of endogenous stimulation of endocannabinoid receptors. ${ }^{19-21} \mathrm{CBD}$ has the capacity to interact with an FXS-compromised endocannabinoid system. Indeed, deletion of FMRP within a mouse model of FXS led to reduced production of 2-AG, decreasing activation of $\mathrm{CB}_{1}$ receptors in the central nervous sys- tem. ${ }^{20} \mathrm{CBD}$ has been shown to increase 2-AG availability, ${ }^{22}$ potentially attenuating or reversing one of the biological mechanisms of abnormal cellular function in FXS. ${ }^{20}$ Importantly, $\mathrm{CB}_{1}$ protein expression appears unaffected in FMR1 knockout (KO) mice, suggesting that the downstream elements of endocannabinoid signaling can be engaged, even in the absence of FMRP. ${ }^{19}$

In addition to the role of $2-\mathrm{AG}$, recent work has begun to highlight the potential importance of AEA in addressing social impairment as well as deficits in learning and memory among those with FXS. In an FMR1 KO mouse model of FXS, Qin et al. demonstrated that increased levels of AEA were associated with greater cognitive performance. ${ }^{23}$ Similarly, Wei et al. utilized mouse models of FXS to show that AEA-mediated signaling at $\mathrm{CB}_{1}$ receptors, driven by oxytocin, controls social reward ${ }^{24}$ and that increasing AEA activity resulted in reductions in social impairment. ${ }^{25}$ Much like its impact on 2-AG, CBD has been shown to increase levels of AEA by binding to fatty acid-binding proteins, which transport AEA to the catabolic enzyme fatty acid amide hydrolase, an enzyme that breaks down AEA. ${ }^{26-28}$ Binding to fatty acidbinding proteins is thought to increase AEA availability and $\mathrm{CB}_{1}$ activation.

\section{CBD and FXS: Other Mechanisms of Action}

The mechanisms underlying the potential benefits of CBD for FXS span far beyond the endocannabinoid system. Beyond providing benefit to patients with FXS through increases in 2-AG and AEA availability, CBD may positively affect synaptic plasticity. Studies of FMR1 KO mice have identified discrete alterations in synaptic plasticity in specific brain regions, including an increase in long-term depression (LTD) in the hippocampus, ${ }^{23,29}$ and preclinical data suggest that reducing LTD in FMR1 KO mice requires activation of endocannabinoid receptors. ${ }^{19}$ Therefore, it is hypothesized that CBD may increase synaptic plasticity in FXS, facilitating one of the basic cellular mechanisms thought to be associated with learning and improvements in cognition. ${ }^{29}$

More recent work has also begun to identify deficits in GABA receptor expression among those with FXS. As FMRP has been shown in animal models to enhance expression of GABA receptors, the lack of FMRP among those with FXS has been associated with fewer GABA receptors. ${ }^{30}$ Indeed, preclinical studies in FMR1 KO mice have consistently shown downregulation of the GABA system. ${ }^{11}$ As CBD acts as a positive 
allosteric modulator of GABA-A receptors, ${ }^{31} \mathrm{CBD}$ may also act to enhance the binding affinity for GABA.

The impact of CBD on serotonin represents a mechanism by which CBD may aid in reduction of social anxiety and resulting avoidance experienced by patients with FXS. Indeed, the anxiolytic effects of CBD have been reported in over 30 preclinical studies, using multiple models of anxiety (i.e., generalized anxiety, stress-induced anxiety, panic disorder, and contextual fear conditioning), ${ }^{32,33}$ as well as in a growing number of human studies, including within-social anxiety. ${ }^{32,34}$ Several preclinical studies have identified the serotonin $1 \mathrm{~A}$ receptor as one mechanism through which CBD exerts its anxiolytic effects. ${ }^{35,36}$

\section{Case Reports}

We report the cases of three patients with diagnosed FXS who received oral forms of botanically derived $\mathrm{CBD}+$ solutions and reported clinical symptom improvement.

\section{Patient 1}

Patient 1 is a 3-year 6-month-old male with FXS diagnosed at age 15 months with 250 to 650 CGG repeats. He was born full-term following an uncomplicated pregnancy and delivery at seven pounds six ounces. In the newborn period, he began having problems latching for breastfeeding, and he was fed pumped breast milk and formula from a bottle; he had frequent gagging and spitting up. By 6 months of age, with introduction of soft baby foods, he was often dysphagic, choked easily, and was intolerant of chunky or textured foods. Testing for food allergies was negative, and his formula was changed multiple times over his first year of life. He grew slowly at the third to fifth percentile for weight and the 50th percentile for length until 15 months of age. He was also hypotonic with delays in gross motor skills. He had behavioral concerns, including atypical motor movements, frequent repetitive moving, stiffening and shaking of his legs, body rocking, and repetitive finger stereotypies while touching his ears. He displayed difficulty adjusting to new or noisy places and changes in routine, as well as trouble making eye contact and a short attention span during play and in social interactions; he would sometimes stare off and seem disconnected for 10 to $30 \mathrm{sec}$.

At 15 months of age, he was evaluated and underwent a brain magnetic resonance imaging (MRI), electroencephalography (EEG), creatine kinase, plasma amino acids, and thyroid and genetic testing. His
MRI, EEG, microarray, and laboratory parameters were normal. His FXS DNA test results showed a full mutation for FXS with 260 to 650 fully methylated CGG repeats.

At this time, the patient's parents independently obtained and began administering an oral paste comprising $18 \%$ to $23.5 \% \mathrm{CBD}$ and trace amounts $(0.03 \%)$ of delta-9-tetrahydrocannabinol (THC) that delivered $50 \mathrm{mg}$ of CBD per day (RSHO ${ }^{\mathrm{TM}}$ Blue Label; Naturewell, Inc., San Diego, CA). They combined the CBD paste with coconut oil, heated the mixture until it liquefied, and then used a syringe to administer the liquid preparation orally. During the first month of $\mathrm{CBD}+$ treatment, the family noticed behavioral improvements.

After 1 month of CBD+ monotherapy, the then 16month-old patient began specialized care in the form of speech and language therapy and occupational therapy for FXS. A baseline measure of adaptive functioning was obtained (Table 1). Over the next 3 months of daily 50-mg CBD+ treatment with adjunctive therapy, the family noted continued improvements in a wide range of clinical parameters. Feeding improved markedly with increased willingness to eat solid foods and increased intake overall. His parents also noted improvements in motor coordination, more frequent vocalizations, less rocking and kicking during feeds, more frequent and longer eye contact, an increase in positive interactions with other children, greater willingness to explore new places, and less self-stimulatory behavior. Many of the parental observations were also confirmed by the patient's occupational and speech therapists, some of whom were unaware of the initiation of $\mathrm{CBD}+$ treatment. $\mathrm{CBD}+$ solution was the only medication used in treatment during this time.

Table 1. Patient 1: Adaptive Functioning ${ }^{a}$ at 1 Year 4 Months of Age and 3 Years 0 Months of Age

\begin{tabular}{|c|c|c|c|c|}
\hline & \multicolumn{2}{|c|}{1 year 4 months } & \multicolumn{2}{|c|}{3 years 0 months } \\
\hline & $\begin{array}{l}\text { Standardized } \\
\text { score }^{b}\end{array}$ & Percentile & $\begin{array}{l}\text { Standardized } \\
\text { score }^{b}\end{array}$ & Percentile \\
\hline Communication & 84 & 14th & 87 & 19th \\
\hline Daily living skills & 71 & $3 r d$ & 77 & 6th \\
\hline Socialization & 86 & 18th & 79 & 8th \\
\hline Motor skills & 78 & 7th & 70 & 2nd \\
\hline $\begin{array}{l}\text { Adaptive behavior } \\
\text { composite }\end{array}$ & 76 & 5 th & 75 & 5 th \\
\hline
\end{tabular}

aineland adaptive behavior scales, second edition-parent survey.

${ }^{\mathrm{b}}$ Mean $\pm \mathrm{SD}=100 \pm 15$.

$\mathrm{SD}$, standard deviation. 
At the 4-month follow-up visit, the patient's weight had increased to the 15th percentile, while his length remained at the 50th percentile. Developmental progress included improved fine and gross motor skills, decreased repetitive rocking, improved social interest/ engagement, improved vocalizations, and decreased hyperactivity. The patient began a regimen of sertraline $(2.5 \mathrm{mg}$ per day) due to preliminary evidence of improved language and development in young children with FXS treated with sertraline. ${ }^{37,38}$ Subsequent follow-up visits (at 21 and 27 months of age) showed continued improvement in language and developmental skills. The patient started walking and self-feeding independently, exhibited more frequent and varied vocalizations, fewer repetitive and motor behaviors, fewer sensory sensitivities (e.g., tolerated noise and lights of fireworks), and more frequent initiation of joint attention with pointing. Weight increased to the 25 th percentile. He continued using CBD+ solution and remained in a strong early intervention program: $1 \mathrm{~h}$ per week of occupational, speech, and physical therapy; $6 \mathrm{~h}$ per week of Early Start Denver Model behavioral therapy and applied behavioral analysis.

When the patient was 30 months of age, his parents chose to discontinue $\mathrm{CBD}+$ treatment to instead explore minocycline, shown to improve behavioral symptoms in some patients with FXS. ${ }^{39}$ The initiation of $25 \mathrm{mg}$ per day of minocycline coupled with cessation of $\mathrm{CBD}+$ treatment resulted in increased anxiety, more frequent meltdowns, and more difficulty falling and maintaining sleep.

During a clinic visit when the patient was 3 years of age, it was noted that the patient continued to engage and make slow progress, although anxiety, frequent meltdowns, and difficulties with sleep persisted, and increasing challenges with transitions and attention span were observed. The patient's medical treatments included a daily regimen of $4 \mathrm{mg}$ of sertraline and $25 \mathrm{mg}$ of minocycline. He also continued to receive intensive early intervention services (as described above). At 3 years of age, an updated adaptive behavior assessment was obtained (Table 1). After discussion with his family and medical team, the patient was restarted on $\mathrm{CBD}+$ treatment, $50 \mathrm{mg}$ per day. Following reinitiation of $\mathrm{CBD}+$ treatment, his parents noted reductions in anxiety, fewer meltdowns, and improvements in his ability to fall and stay asleep. The patient has now transitioned into a small preschool where he is continuing to do well and has remained on his CBD+ treatment.

\section{Patient 2}

Patient 2 is a 26-year-old male with full-mutation ( $>200$ CGG repeats) FXS and an IQ score in the mid-50s. Despite a medication regimen that included $60 \mathrm{mg}$ of methylphenidate hydrochloride (once daily), $2.5 \mathrm{mg}$ of aripiprazole, $100 \mathrm{mg}$ of sertraline, $200 \mathrm{mg}$ of minocycline, and $0.2 \mathrm{mg}$ of clonidine (at bedtime), the patient experienced significant symptoms of attention-deficit/ hyperactivity disorder, including hyperactivity, inattention, and impulsivity. He also suffered from anxiety, which led to avoidant behavior and sleep disturbances, and some significant features of autism, including social avoidance, poor eye contact, perseverative behavior, hand stereotypies, and tactile defensiveness.

Due to ongoing symptomatology, the patient began taking a liquid preparation containing $63.9 \% \mathrm{CBD}$, $4 \%$ cannabichromene, $3.4 \%$ THC, and $0.7 \%$ phenylbiguanide, delivered orally by a $1-\mathrm{mL}$ syringe. The patient's parents obtained the solution independent of their physician. Between 0.05 and $0.1 \mathrm{~mL}$ (i.e., 32.0$63.9 \mathrm{mg} \mathrm{CBD}$ ) of the oral solution was delivered in the morning, once per day for 6 weeks, during which time other therapies remained unchanged.

During the first week of treatment with the CBD+ solution, the patient's family noticed that his anxiety level was reduced and he was able to explore and participate in more activities with less social avoidance. His facility with language increased, as shown by greater capacity to engage in longer more meaningful conversations. The quality and duration of his sleep also improved; he no longer awoke and wandered in the middle of the night. His symptoms of anxiety and linguistic skills continued to improve over the 6-week course of treatment with the $\mathrm{CBD}+$ solution. His parents have continued him on a stable dose of $0.1 \mathrm{~mL}$ daily now for 2 years and have observed sustained symptom improvement.

\section{Patient 3}

Patient 3 is a 22 -year-old female who was diagnosed with FXS at 9 years of age with a full mutation of $>200$ CGG repeats. She met all of her early milestones appropriately, but at around 3 to 4 years of age, she developed quite significant anxiety and panic attacks. There was mild benefit associated with initiation of psychological therapies, although her mother described difficulties with new settings, social anxiety, frequent negative perseverative thoughts, and ongoing panic attacks that would vary in frequency depending on her routine and social supports. 
Starting in the fourth grade, she had mild academic challenges that were supported with a 504 educational plan through the end of high school. Ongoing anxiety was present and trials of St. John's Wort and other supplements were minimally helpful. She did not receive any other medication treatment. She graduated from college with academic supports, with continued periods of significant anxiety symptoms, social withdrawal, and panic attacks. After college, she began work as an educational aide in a public school, with an exacerbation of anxiety due to this transition into adulthood and pressures of increased independence.

At 22 years of age, her parents provided her with a liquid formulation of hemp oil containing $\sim 43 \mathrm{mg}$ of CBD daily (Charlotte's Web ${ }^{\mathrm{TM}}$ Everyday Advanced; CWB Holdings, Inc., Denver, CO), which she began taking. She described feeling calmer, with fewer perseverative worries, and a cessation of panic attacks. This led to more interactions and activities with peers and improved performance at work. Her mother also noted that she became more engaged socially, calmed more easily when frustrated, and was less likely to fixate on negative aspects of various situations. She missed about 1 week of dosing when on a family vacation out of town, and after a few days without treatment, she experienced recurrence of anxiety symptoms and reemergence of panic attacks. These resolved after she was home and able to restart her CBD+ treatment. She is currently working full time and living independently. She has continued CBD+ treatment for two and a half years with sustained therapeutic benefit.

\section{Discussion}

Based on its morbidity and prevalence, its association with a number of co-occurring problems, such as seizures, anxiety, and sleep disturbances, ${ }^{13}$ and the paucity of efficacious therapeutic options, FXS represents an important public health problem. ${ }^{6}$ The present article provides a brief review of recent research that documents the promise of CBD as a therapeutic agent for patients with FXS. Also described are the cases of one pediatric and two adult patients with FXS for whom $\mathrm{CBD}+$ treatment appears to have contributed to positive changes in anxiety and/or language skills, with no observed adverse events.

Before starting $\mathrm{CBD}+$ treatment, Patient 1 experienced heightened symptoms of anxiety, frequent tantrums, and sleep difficulties. Over the first month of $\mathrm{CBD}+$ monotherapy, and subsequent 3 months of $\mathrm{CBD}+$ treatment combined with speech, language, and occupational therapy, the patient made considerable progress with feeding and weight gain, exhibited better oral-motor coordination, had decreased social avoidance and sensory sensitivities, and showed improvements in attention span/engagement, the frequency and severity of atypical motor movements, and general level of hyperactivity. Upon discontinuation of CBD+ treatment, the patient's prior symptoms reemerged. While a strong therapy program and later addition of other medications likely contributed to this patient's overall developmental progress, the temporally related improvements in anxiety, feeding, tantrums, and sleep-evident when CBD+ treatment was initially started as monotherapy and then reinstated following cessation-are compelling support for the benefits of CBD+ treatment for this patient. By maintaining his adaptive functioning scores from 1 to 3 years of age, the patient demonstrated a significant improvement over the characteristic developmental trajectory of young male children with FXS, where a decrease in adaptive functioning scores is typically observed between 2 and 6 years of age. ${ }^{40}$

Patient 2 showed a similarly encouraging response to $\mathrm{CBD}+$ treatment, experiencing reduced anxiety, improved use of language, and better sleep within 1 week of beginning treatment with a $\mathrm{CBD}+$ solution. It is also remarkable that the patient continued to demonstrate symptom improvement over the initial 6-week treatment period, with longer-term follow-up highlighting continued use of $\mathrm{CBD}+$ solution with sustained benefit.

In Patient 3, the use of CBD+ solution was also associated with a positive effect in a higher functioning female with FXS and long-standing anxiety symptoms. Similar to Patient 1, treatment discontinuation was associated with a recurrence of anxiety symptoms, with reinitiation of $\mathrm{CBD}+$ treatment leading to symptom improvement and resulting long-term use.

The present findings, coupled with the available preclinical data, highlight the potential for CBD as an intervention for individuals with FXS. The existing literature combines to demonstrate that CBD may positively impact individuals with FXS through many mechanisms, including the endocannabinoid system, GABA, and serotonin. While a number of drugs have been developed to target specific systems (e.g., GABA agonists), CBD has the potential to yield a multifaceted benefit to individuals with FXS due to its multiple mechanisms of action. CBD has not only been shown to be generally well tolerated relative to other treatments 
used in this population, ${ }^{41}$ but also numerous studies have documented its benefits in terms of sleep quality, ${ }^{42,43}$ anxiety (including social anxiety), ${ }^{34,44}$ and cognitive impairment ${ }^{45}$-symptoms experienced by the individuals profiled in the present case series.

These data serve as stepping stones upon which proof-of-concept open-label trials should be based. As with many patients, however, those discussed herein used orally administered botanical CBD+ solution that is not regulated by the Food and Drug Administration and, thus, inconsistencies in availability, quality, purity, and labeling ${ }^{46,47}$ make research, interpretations, and clinical recommendations challenging. The present case series is limited by its reliance on manufacturerreported cannabinoid content as well as the lack of multimethod assessment of patient symptomatology, including clinimetric data. The observed clinical benefit of CBD+ treatment in case studies, particularly with respect to caregiver-reported behavioral outcomes, must also be interpreted with caution given the significant placebo effects that have been documented in clinical trials of $\mathrm{CBD} .{ }^{48}$ Only placebo-controlled trials will be able to elucidate the true therapeutic effects of CBD/ $\mathrm{CBD}+$ treatment on FXS symptomatology. Until rigorous clinical trials have demonstrated the efficacy of $\mathrm{CBD} / \mathrm{CBD}+$ treatment for FXS, current treatments for the many behavioral problems associated with FXS should be utilized before off-label use of CBD+ products.

In an effort to overcome existing limitations, future studies should independently test patient samples to confirm actual constituents of each $\mathrm{CBD}+$ preparation $^{47}$ and utilize well-validated caregiver-reported assessments of anxiety and other FXS symptomatology, in addition to unstructured caregiver- and clinicianbased reports, in an attempt to more completely track each patient's course while in clinical care. Due to inconsistencies observed in many oral botanical preparations, rigorous examinations of pharmaceutical-grade preparations of CBD should be explored as potential treatments for children and adolescents with FXS.

\section{Acknowledgment}

Writing services for some components of the manuscript were provided by Christopher Caiazza.

\section{Author Disclosure Statement}

N.T. has received funding from Neuren, Alcobra, Roche, and Seaside to carry out treatment trials in FXS. She has also consulted with Novartis, Zynerba, and Ovid regarding treatment in FXS. M.B.M. is an employee of Zynerba Pharmaceuticals. R.H. has received funding from Novartis, Marinus, Neuren, Alcobra, Seaside, and Roche to carry out treatment trials in FXS. She has also consulted with Fulcrum, Zynerba, and Ovid regarding treatment in FXS.

\section{References}

1. Hagerman RJ, Berry-Kravis E, Hazlett HC, et al. Fragile X syndrome. Nat Rev Dis Primers. 2017;3:17065

2. Darnell JC, Richter JD. Cytoplasmic RNA-binding proteins and the control of complex brain function. Cold Spring Harb Perspect Biol. 2012;4: a012344.

3. Turner G, Webb T, Wake $S$, et al. Prevalence of fragile $X$ syndrome. Am J Med Genet. 1996;64:196-197.

4. Coffee $B$, Keith $K$, Albizua I, et al. Incidence of fragile $X$ syndrome by newborn screening for methylated FMR1 DNA. Am J Hum Genet. 2009;85: 503-514.

5. Hunter J, Rivero-Arias O, Angelov A, et al. Epidemiology of fragile $X$ syndrome: a systematic review and meta-analysis. Am J Med Genet A. 2014; 164a:1648-1658.

6. Crawford DC, Acuna JM, Sherman SL. FMR1 and the fragile $X$ syndrome: human genome epidemiology review. Genet Med. 2001;3:359-371.

7. Tassone F, Hagerman RJ, Taylor AK, et al. Elevated levels of FMR1 mRNA in carrier males: a new mechanism of involvement in the fragile- $X$ syndrome. Am J Hum Genet. 2000;66:6-15.

8. Garber KB, Visootsak J, Warren ST. Fragile X syndrome. Eur J Hum Genet. 2008;16:666-672.

9. Farzin F, Perry H, Hessl D, et al. Autism spectrum disorders and attentiondeficit/hyperactivity disorder in boys with the fragile $X$ premutation. J Dev Behav Pediatr. 2006;27(2 Suppl):S137-S144.

10. Leigh MJS, Hagerman RJ, HessI D. Fragile X Syndrome. In: Hansen RL, Rogers SJ, eds. Autism and other neurodevelopmental disorders. American Psychiatric Publishing: Arlington, 2013, pp. 57-76.

11. Lozano R, Hare EB, Hagerman RJ. Modulation of the GABAergic pathway for the treatment of fragile $X$ syndrome. Neuropsychiatr Dis Treat. 2014;10: 1769-1779.

12. Fragile $X$ Clinical and Research Consortium. Consensus of the Fragile $X$ Clinical \& Research Consortium on Clinical Practices. Medications for Individuals with Fragile X Syndrome. Available at: https://fragilex.org// wp-content/uploads/2012/08/ Medications_for_Individuals_with_Fragile_X_Syndrome2012-Oct.pdf. Accessed April 11, 2018.

13. Lozano R, Azarang A, Wilaisakditipakorn T, et al. Fragile X syndrome: a review of clinical management. Intractable Rare Dis Res. 2016;5:145-157.

14. Karhson DS, Krasinska KM, Dallaire JA, et al. Plasma anandamide concentrations are lower in children with autism spectrum disorder. Mol Autism. 2018;9:18.

15. Mouslech Z, Valla V. Endocannabinoid system: an overview of its potential in current medical practice. Neuro Endocrinol Lett. 2009;30:153-179.

16. Pacher $P$, Batkai $S$, Kunos $G$. The endocannabinoid system as an emerging target of pharmacotherapy. Pharmacol Rev. 2006;58:389-462.

17. Castillo PE, Younts TJ, Chavez AE, et al. Endocannabinoid signaling and synaptic function. Neuron. 2012;76:70-81.

18. Ohno-Shosaku T, Kano M. Endocannabinoid-mediated retrograde modulation of synaptic transmission. Curr Opin Neurobiol. 2014;29:1-8.

19. Zhang L, Alger BE. Enhanced endocannabinoid signaling elevates neuronal excitability in fragile X syndrome. J Neurosci. 2010;30:5724-5729.

20. Jung KM, Sepers $M$, Henstridge $C M$, et al. Uncoupling of the endocannabinoid signalling complex in a mouse model of fragile $\mathrm{X}$ syndrome. Nat Commun. 2012;3:1080.

21. Maccarrone M, Rossi S, Bari M, et al. Abnormal mGlu 5 receptor/endocannabinoid coupling in mice lacking FMRP and BC1 RNA. Neuropsychopharmacology. 2010;35:1500-1509.

22. Maione S, Piscitelli F, Gatta L, et al. Non-psychoactive cannabinoids modulate the descending pathway of antinociception in anaesthetized rats through several mechanisms of action. Br J Pharmacol. 2011;162: 584-596. 
23. Qin M, Zeidler Z, Moulton K, et al. Endocannabinoid-mediated improvement on a test of aversive memory in a mouse model of fragile $X$ syndrome. Behav Brain Res. 2015;291:164-171.

24. Wei $D$, Lee $D, C o x C D$, et al. Endocannabinoid signaling mediates oxytocin-driven social reward. Proc Natl Acad Sci U S A. 2015;112:1408414089.

25. Wei D, Dinh D, Lee D, et al. Enhancement of anandamide-mediated endocannabinoid signaling corrects autism-related social impairment. Cannabis Cannabinoid Res. 2016;1:81-89.

26. Elmes MW, Kaczocha M, Berger WT, et al. Fatty acid-binding proteins (FABPs) are intracellular carriers for Delta9-tetrahydrocannabinol (THC) and cannabidiol (CBD). J Biol Chem. 2015;290:8711-8721.

27. Pertwee RG. The diverse $C B 1$ and $C B 2$ receptor pharmacology of three plant cannabinoids: delta9-tetrahydrocannabinol, cannabidiol and delta9-tetrahydrocannabivarin. Br J Pharmacol. 2008;153:199-215.

28. Leweke FM, Piomelli D, Pahlisch F, et al. Cannabidiol enhances anandamide signaling and alleviates psychotic symptoms of schizophrenia. Transl Psychiatry. 2012;2:e94.

29. Huber KM, Gallagher SM, Warren ST, et al. Altered synaptic plasticity in a mouse model of fragile $X$ mental retardation. Proc Natl Acad Sci U S A. 2002;99:7746-7750.

30. Lozano R, Martinez-Cerdeno V, Hagerman RJ. Advances in the understanding of the gabaergic neurobiology of FMR1 expanded alleles leading to targeted treatments for Fragile $X$ Spectrum disorder. Curr Pharm Des. 2015;21:4972-4979.

31. Bakas T, van Nieuwenhuijzen PS, Devenish SO, et al. The direct actions of cannabidiol and 2-arachidonoyl glycerol at GABAA receptors. Pharmacol Res. 2017;119:358-370.

32. Blessing EM, Steenkamp MM, Manzanares J, et al. Cannabidiol as a potential treatment for anxiety disorders. Neurotherapeutics. 2015;12: 825-836.

33. Loflin MJ, Babson KA, Bonn-Miller MO. Cannabinoids as therapeutic for PTSD. Curr Opin Psychol. 2017;14:78-83.

34. Bergamaschi MM, Queiroz $\mathrm{RH}$, Chagas $\mathrm{MH}$, et al. Cannabidiol reduces the anxiety induced by simulated public speaking in treatment-naïve social phobia patients. Neuropsychopharmacology. 2011;36:1219-1226.

35. Campos AC, Guimaraes FS. Involvement of 5HT1A receptors in the anxiolytic-like effects of cannabidiol injected into the dorsolateral periaqueductal gray rats. Psychopharmacology. 2008;199:223-230.

36. Norris C, Loureiro M, Kramar C, et al. Cannabidiol modulates fear memory formation through interactions with seratonergic transmission in the mesolimbic system. Neuropsychopharmacology. 2016;41:2839-2850.

37. Indah Winarni T, Chonchaiya W, Adams E, et al. Sertraline may improve language developmental trajectory in young children with fragile $x$ syndrome: a retrospective chart review. Autism Res Treat. 2012;2012:104317.

38. Greiss Hess L, Fitzpatrick SE, Nguyen DV, et al. A randomized, doubleblind, placebo-controlled trial of low-dose sertraline in young children with Fragile X Syndrome. J Dev Behav Pediatr. 2016;37:619-628.

39. Leigh MJ, Nguyen DV, Mu Y, et al. A randomized double-blind, placebocontrolled trial of minocycline in children and adolescents with fragile $x$ syndrome. J Dev Behav Pediatr. 2013;34:147-155.
40. Klaiman C, Quintin EM, Jo B, et al. Longitudinal profiles of adaptive behavior in fragile $X$ syndrome. Pediatrics. 2014;134:315-324.

41. Rohleder C, Muller JK, Lange B, et al. Cannabidiol as a potential new type of an antipsychotic. A critical review of the evidence. Front Pharmacol. 2016;7:422.

42. Carlini EA, Mechoulam R, Lander N. Anticonvulsant activity of four oxygenated cannabidiol derivatives. Res Commun Chem Pathol Pharmacol. 1975;12:1-15.

43. Chagas MH, Eckeli AL, Zuardi AW, et al. Cannabidiol can improve complex sleep-related behaviours associated with rapid eye movement sleep behaviour disorder in Parkinson's disease patients: a case series. J Clin Pharm Ther. 2014;39:564-566.

44. Viveros MP, Marco EM, File SE. Endocannabinoid system and stress and anxiety responses. Pharmacol Biochem Behav. 2005;81:331-342.

45. Osborne AL, Solowij N, Weston-Green K. A systematic review of the effect of cannabidiol on cognitive functoin: relevance to schizophrenia. Neurosci Biobehav Rev. 2017;72:310-324.

46. Mead A. The legal status of cannabis (marijuana) and cannabidiol (CBD) under U.S. law. Epilepsy Behav. 2017;70(Pt B):288-291.

47. Bonn-Miller MO, Loflin MJE, Thomas BF, et al. Labeling accuracy of cannabidiol extracts sold online. JAMA. 2017;318:1708-1709.

48. Thiele EA, Marsh ED, French JA, et al. Cannabidiol in patients with seizures associated with Lennox-Gastaut syndrome (GWPCARE4): a randomized, double-blind, placebo-controlled phase 3 trial. Lancet. 2018;391:10851096.

Cite this article as: Tartaglia N, Bonn-Miller M, Hagerman R (2019)

Treatment of fragile $X$ syndrome with cannabidiol: a case series study and brief review of the literature, Cannabis and Cannabinoid Research 4:1 3-9, DOI: 10.1089/can.2018.0053.

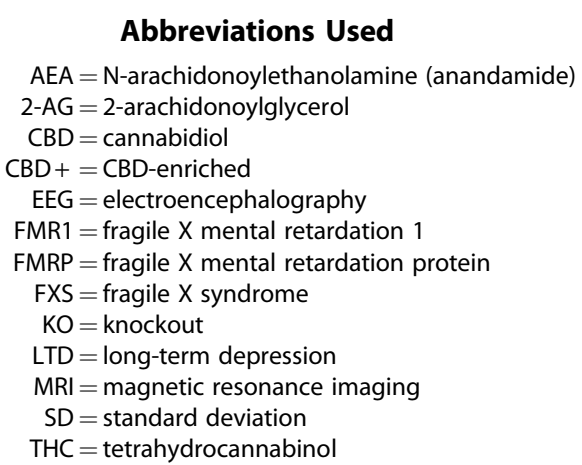

